\title{
BMC
}

Public Health
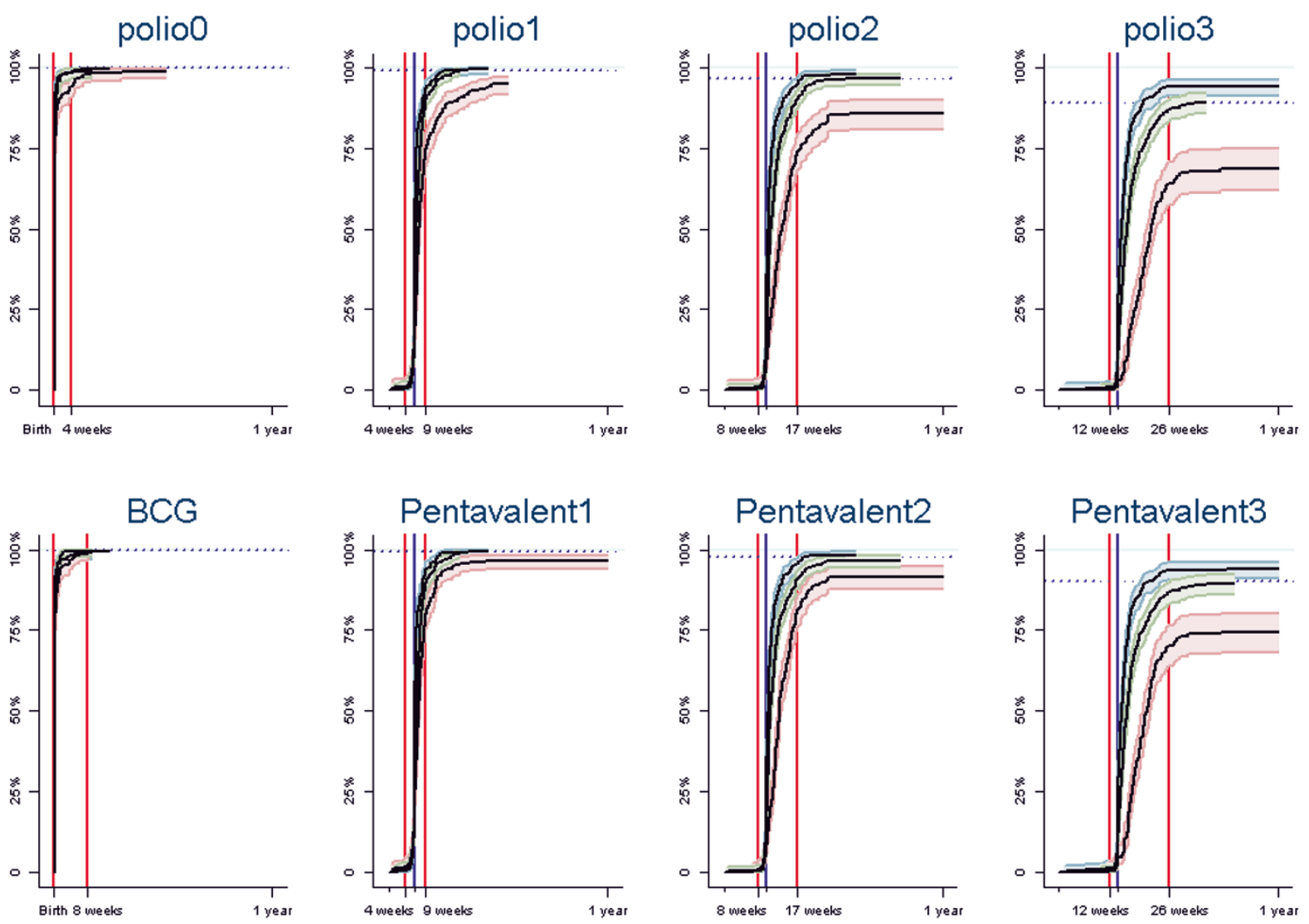

\section{Vaccination coverage and timeliness in three South African areas: a prospective study}

Fadnes et al. 


\title{
Vaccination coverage and timeliness in three South African areas: a prospective study
}

\author{
Lars T Fadnes ${ }^{1 *}$, Debra Jackson², Ingunn MS Engebretsen ${ }^{1}$, Wanga Zembe ${ }^{3}$, David Sanders ${ }^{2}$, Halvor Sommerfelt ${ }^{1,4}$ \\ and Thorkild Tylleskär ${ }^{1}$ for the PROMISE-EBF Study Group
}

\begin{abstract}
Background: Timely vaccination is important to induce adequate protective immunity. We measured vaccination timeliness and vaccination coverage in three geographical areas in South Africa.

Methods: This study used vaccination information from a community-based cluster-randomized trial promoting exclusive breastfeeding in three South African sites (Paarl in the Western Cape Province, and Umlazi and Rietvlei in KwaZulu-Natal) between 2006 and 2008. Five interview visits were carried out between birth and up to 2 years of age (median follow-up time 18 months), and 1137 children were included in the analysis. We used Kaplan-Meier time-to-event analysis to describe vaccination coverage and timeliness in line with the Expanded Program on Immunization for the first eight vaccines. This included Bacillus Calmette-Guérin (BCG), four oral polio vaccines and 3 doses of the pentavalent vaccine which protects against diphtheria, pertussis, tetanus, hepatitis B and Haemophilus influenzae type B.
\end{abstract}

Results: The proportion receiving all these eight recommended vaccines were $94 \%$ in Paarl (95\% confidence interval [Cl] 91-96), 62\% in Rietvlei $(95 \% \mathrm{Cl} 54-68)$ and $88 \%$ in Umlazi (95\%Cl 84-91). Slightly fewer children received all vaccines within the recommended time periods. The situation was worst for the last pentavalent- and oral polio vaccines. The hazard ratio for incomplete vaccination was 7.2 (95\%Cl 4.7-11) for Rietvlei compared to Paarl.

Conclusions: There were large differences between the different South African sites in terms of vaccination coverage and timeliness, with the poorer areas of Rietvlei performing worse than the better-off areas in Paarl. The vaccination coverage was lower for the vaccines given at an older age. There is a need for continued efforts to improve vaccination coverage and timeliness, in particular in rural areas.

Trial registration number: ClinicalTrials.gov: NCT00397150

\section{Background}

Timely vaccination is important to induce adequate immunity [1-3]. Delayed immunisation is a risk factor for pertussis, measles and Haemophilus influenzae type B disease $[1,2,4,5]$. Late administration of the Bacillus Calmette-Guérin (BCG) vaccine is also associated with reduced survival [6].

Some studies have shown that good vaccination coverage for individual vaccines does not necessarily imply timely vaccination [3,7-11]. There may also be non-specific effects of vaccines that can be influenced by timeliness, with potential negative consequences of delayed

\footnotetext{
* Correspondence: Lars.Fadnes@cih.uib.no

${ }^{1}$ Centre for International Health, University of Bergen, Norway

Full list of author information is available at the end of the article
}

immunisation [12,13]. Thus, it is important to take timeliness into account, as relying only on vaccination status can lead to a false assumption of adequate vaccination.

Although some studies have evaluated timely vaccination of some selected vaccines, we are only aware of two published studies from the United States and Uganda where the timeliness of all nationally recommended vaccines has been evaluated $[10,11]$. Only two other studies have assessed timely vaccination for some selected vaccines in African settings [9,14]. There also seems to be conflicting information in South Africa between the nationally reported morbidity related to vaccine preventable diseases compared to reports about large measles outbreaks $[15,16]$. In this study, we assessed immunisation timeliness and vaccination coverage for the first

\section{Biomed Central}

(c) 2011 Fadnes et al; licensee BioMed Central Ltd. This is an Open Access article distributed under the terms of the Creative Commons Attribution License (http://creativecommons.org/licenses/by/2.0), which permits unrestricted use, distribution, and reproduction in any medium, provided the original work is properly cited. 
eight vaccines of the Expanded Program on Immunization (EPI) in South Africa. This included BCG (Bacillus Calmette-Guérin), four doses of oral polio vaccines and three doses of the pentavalent vaccine protecting against diphtheria, pertussis, tetanus, hepatitis B and Haemophilus influenzae type B disease.

\section{Methods}

This study used vaccination information from the community-based cluster-randomized PROMISE-EBF trial promoting exclusive breastfeeding by peer-counsellors in three South African sites between 2006 and 2008 (ClinicalTrials.gov no: NCT00397150) [17]. A total of 34 clusters from three separate areas in South Africa were chosen: Paarl in Western Cape Province (peri-urban), and Umlazi (peri-urban) and Rietvlei (rural) in KwaZulu-Natal Province. The infant mortality rate (IMR) in Paarl is about 40/1000, and the antenatal HIV-prevalence was $10 \%$ at the time of the study [18]. The IMR in Umlazi was around 60/1000 and the antenatal HIV-prevalence $42 \%$. The poor and rural area Rietvlei had an IMR of 99/1000 and an antenatal HIV-prevalence of $34 \%$ at the time of the study.

Some of the, mothers approached for study participation in the PROMISE-EBF study were excluded due to an intention to formula feed (142). They were enrolled in the Good start Study [19]. Both the participants in the PROMISE-EBF study and the Good Start Study provided information on vaccination. A total of 1276 mother-infant pairs were recruited from both of the studies. Among these, 139 were excluded due to relocation or being lost-to-follow-up, twin delivery, death of the infant or mother before 3 weeks after birth, or severe malformations (additional file 1, figure S1). Thus, 1137 mother-infant pairs remained in the analysis. The mother-infant pairs were scheduled to be interviewed at $3,6,12$ and 24 weeks after birth, with an additional follow-up interview between 18 and 24 months of age. Most vaccination information was collected during the last two visits. Vaccination information was collected from the last two visits. A health card was seen for 935 (98\%) among those who were interviewed at 24 weeks when much of the vaccination information was gathered. These health cards were dated, but in some instances the date was invalid. The median follow-up time was 18 months.

\section{Data management}

Data was collected through interviews by trained data collectors, and subsequently entered using EpiData (http://www.epidata.dk). Data analysis was done with Stata version SE11.1 (Stata Corporation).

\section{Vaccination timeliness and coverage}

The Expanded Program on Immunization in South Africa recommended the following vaccines to be given at specific ages during the study period in line with the recommendations from the World Health Organisation (time ranges given in parentheses) [9,20]:

O The first vaccination visit is at birth when the BCG (birth-8 weeks) and oral polio (birth-4 weeks) vaccines are given.

$\circ$ The following three vaccination visits include the oral polio vaccine and a pentavalent vaccine which protects against diphtheria, tetanus and pertussis (DTP), Haemophilus influenzae type B (Hib) disease and hepatitis $\mathrm{B}(\mathrm{HBV})$. The first dose is given at 6 weeks ( 4 weeks- 2 months), then again at 10 weeks ( 8 weeks- 4 months), and at 14 weeks (12 weeks- 6 months).

○ The measles vaccine is given at 9 months (38 weeks-12 months).

○ At 18 months, a booster dose was given with protection against measles, polio, diphtheria, pertussis and tetanus. The recommended time range of the booster vaccination has not been clearly defined.

- In 2009 (after completed data collection), a rotavirus and a 7 -valent pneumococcal vaccine were added to the program.

Vaccines given outside these ranges will be referred to as untimely vaccinations.

\section{Analysis}

With the exceptions of measles and booster vaccines, vaccination timeliness was analysed with Kaplan-Meier time-to-event analysis in line with Laubereau et al. [21]. Coverage was determined at the end of follow-up. Vaccination data were gathered from the children's health cards. When vaccinations were registered without a valid date, we assumed that the age when the children were given the specific vaccines was similar to the age of those who had their vaccinations dated (around 2\% had missing dates for each vaccine). The confidence intervals (CI) were estimated with Greenwood's pointwise method. The timing of measles and booster vaccines is presented with histograms. Due to a question that was missed out in the questionnaire, we had to make an assumption whether the reported timing of the measles vaccine was either the first measles vaccine or the booster vaccine. We assumed that vaccinations received before 12 months of age were the first dose of measles vaccine, and vaccinations after 12 months were booster vaccinations. Due to the structural weaknesses 
in the recording of measles vaccination in this study, the information on measles vaccination must be interpreted with care.

To investigate determinants of timely vaccination, we used a cluster adjusted Cox regression analysis. The Cox regression assumption of proportional hazards was checked with Schoenfeld residuals, both graphically and with a significance test. Tied cases were handled with the exact partial-likelihood method. Rational interactions were evaluated. Log linearity was checked by plotting of Martingale residuals for the complete model vs. a model with one omitted variable. We present univariable and multivariable models for both coverage at the end of follow-up and timeliness. The multivariable analyses used a stepwise selection with removal of covariates when $\mathrm{p}>$ 0.1 . The models were adjusted for cluster.

Socioeconomic wealth index was constructed with the use of multiple correspondence analysis based on ownership of assets as mobile phone and television, and house characteristics including water source, roof material and toilet type. This method is analogous to principal component analysis, and better suited for categorical data [22]. The children's families were grouped into quintiles on the basis of socioeconomic rank.

\section{Ethics}

Ethical approval was granted by Ethics Committee of the Medical Research Council South Africa, and Regional Committees for Medical and Health Research Ethics, Western Norway. Signed or thumb-printed informed consent was obtained from each mother prior to study participation.

\section{Results}

There were large variations between the three sites in vaccination coverage and timeliness, both for the individual vaccines and all the first eight vaccines assessed together, table 1, figures 1, 2 and 3 . The timing of the individual vaccines and the timing of all vaccines presented with hazard plot are available as additional files 2, 3, 4, 5, 6, 7, 8, 9 and10 (figures S2, S3, S4, S5, S6, S7, S8, S9 andS10).

The proportion of children receiving all of the first eight recommended vaccines were 94\% in Paarl (95\%CI 9196), $88 \%$ in Umlazi (95\%CI 84-91) and 62\% in Rietvlei (95\%CI 54-68). Fewer children received all vaccines within the recommended time period, $88 \%$ in Paarl (95\% confidence interval 84-91), 79\% in Umlazi (95\%CI 74-82) and 58\% in Rietvlei (95\%CI 51-64). Nearly all untimely vaccinations were given too late.

The coverage was lowest for the last oral polio vaccine with a coverage rate of $87 \%$ (95\%CI $85-89)$ for all sites combined, and for the last pentavalent vaccine with coverage of $88 \%$ (95\%CI $86-90)$. Rietvlei had the lowest coverage also for these vaccines.

Among the children above 12 months of age, 669 (86\%) had received at least one measles vaccine. The time for the measles vaccination was distributed around the recommended time period of 38 weeks, figure 3 . The distribution of booster vaccination was around 18 months with a small amount of scattering.

\section{Determinants of incomplete and untimely vaccination}

The mean level of education among the mothers was 10 years of schooling in Paarl, 11 years in Umlazi and 9 years in Rietvlei. The socioeconomic situation was different in the 3 sites, reflected in 328 (94\%) having electricity in Paarl, 446 (93\%) in Umlazi, and 185 (61\%) in Rietvlei.

No interactions were significant and meaningful in magnitude compared to the individual factors. No combination variables had correlation values above 0.7 . The Cox regression models revealed a strong association between geographical site and both incomplete vaccinated at the end of follow-up and untimely vaccination, table 2. The hazard ratio (HR) for incomplete vaccinated was 7.2 (95\%CI 4.7-11) for Rietvlei compared to Paarl. The association between geographical site and untimely vaccination was slightly weaker, with a HR 3.3 (95\%CI 2.3-4.7) for Rietvlei compared to Paarl.

The children being delivered at home or children with several siblings also had a higher risk of incomplete and untimely vaccination. Socioeconomic factors were also strongly associated with timely vaccination and vaccination coverage in the univariable analysis, but was nearly nullified when including geographical site in the model. A site stratified analysis confirmed that socio-economy within the site was not associated with vaccination coverage in Rietvlei and Umlazi, but an association was seen in Paarl (data not shown).

Breastfeeding peer-counselling was also associated with vaccination coverage, with higher coverage among children receiving peer-counselling on breastfeeding and children of mothers who intended to formula feed.

\section{Discussion}

This study revealed substantial differences in vaccination between the different geographical sites. There was a 7 fold hazard ratio of not being vaccinated at the end of follow-up for Rietvlei compared to Paarl. Rietvlei is a poor and rural area, but adjusting for socio economic or other factors did not alter the association substantially. This is consistent with other studies in these three populations which have shown strong geographic differences in health services quality and health outcomes [19]. 
Table 1 Coverage and timeliness of vaccines in the South African EPI program.

\begin{tabular}{|c|c|c|c|c|c|c|}
\hline & Site & $\begin{array}{c}\text { Coverage } \\
\text { (end of follow-up) }\end{array}$ & Timely vaccination & Given too early & Given too late & $\begin{array}{c}\text { Not given } \\
\text { (at end of follow-up) }\end{array}$ \\
\hline \multirow[t]{4}{*}{ Polio 0} & Paarl & $99(96-100)$ & $99(96-100)$ & $0(0-0)$ & $0(0-0)$ & $1(0-4)$ \\
\hline & Umlazi & $100(98-100)$ & $99(97-100)$ & $0(0-0)$ & $1(0-1)$ & $0(0-2)$ \\
\hline & Rietvlei & $99(96-100)$ & $94(91-97)$ & $0(0-0)$ & $4(3-6)$ & $1(0-4)$ \\
\hline & Total & $100(99-100)$ & 97 (96-98) & $0(0-0)$ & $3(2-3)$ & $0(0-1)$ \\
\hline \multirow[t]{4}{*}{ BCG vaccine } & Paarl & 99 (97-100) & $99(97-100)$ & $0(0-0)$ & $0(0-0)$ & $1(0-3)$ \\
\hline & Umlazi & $100(98-100)$ & $100(98-100)$ & $0(0-0)$ & $0(0-0)$ & $0(0-2)$ \\
\hline & Rietvlei & $99(97-100)$ & $99(97-100)$ & $0(0-0)$ & $0(0-0)$ & $1(0-3)$ \\
\hline & Total & $99(98-100)$ & $99(98-100)$ & $0(0-0)$ & $0(0-0)$ & $1(0-2)$ \\
\hline \multirow[t]{4}{*}{ Polio 1} & Paarl & $100(98-100)$ & $92(90-93)$ & $1(0-2)$ & $7(5-8)$ & $0(0-2)$ \\
\hline & Umlazi & $99(98-100)$ & 88 (86-89) & $1(0-3)$ & $10(8-12)$ & $1(0-2)$ \\
\hline & Rietvlei & $96(92-98)$ & $72(67-75)$ & $1(0-4)$ & $22(19-25)$ & $4(2-8)$ \\
\hline & Total & 99 (98-99) & $85(84-87)$ & $1(1-2)$ & $12(11-14)$ & $1(1-2)$ \\
\hline \multirow[t]{4}{*}{ DPT1+Hib+HBV } & Paarl & $100(98-100)$ & $93(90-94)$ & $0(0-2)$ & $6(4-8)$ & $0(0-2)$ \\
\hline & Umlazi & $99(98-100)$ & $88(86-89)$ & $1(1-3)$ & $10(8-12)$ & $1(0-2)$ \\
\hline & Rietvlei & $97(95-99)$ & 77 (73-79) & $2(1-4)$ & $19(16-21)$ & $3(1-5)$ \\
\hline & Total & $99(98-100)$ & $87(85-88)$ & $1(1-2)$ & $11(10-12)$ & $1(0-2)$ \\
\hline \multirow[t]{4}{*}{ Polio 2} & Paarl & 99 (97-100) & $94(92-94)$ & $1(0-2)$ & $4(3-5)$ & $1(0-3)$ \\
\hline & Umlazi & 98 (96-99) & $90(87-91)$ & $0(0-2)$ & $8(6-9)$ & $2(1-4)$ \\
\hline & Rietvlei & 87 (82-91) & $72(67-75)$ & $1(0-3)$ & $14(12-14)$ & $13(9-18)$ \\
\hline & Total & 96 (94-97) & $87(85-88)$ & $1(0-1)$ & 8 (7-9) & $4(3-6)$ \\
\hline \multirow[t]{4}{*}{ DPT2+Hib+HBV } & Paarl & 99 (97-100) & 95 (93-95) & $0(0-2)$ & $3(2-4)$ & $1(0-3)$ \\
\hline & Umlazi & 98 (96-99) & $90(88-91)$ & $0(0-2)$ & 7 (6-8) & $2(1-4)$ \\
\hline & Rietvlei & 92 (88-95) & 80 (75-82) & $0(0-3)$ & $12(10-13)$ & $8(5-12)$ \\
\hline & Total & 97 (96-98) & $90(88-91)$ & $0(0-1)$ & $7(6-8)$ & $3(2-4)$ \\
\hline \multirow[t]{4}{*}{ Polio 3} & Paarl & 94 (91-96) & 93 (91-94) & $1(0-3)$ & $0(0-0)$ & $6(4-9)$ \\
\hline & Umlazi & 98 (96-99) & 87 (83-88) & $0(0-2)$ & $11(9-13)$ & $2(1-4)$ \\
\hline & Rietvlei & $69(62-75)$ & 64 (57-71) & $0(0-0)$ & $5(4-5)$ & $31(25-38)$ \\
\hline & Total & 87 (85-89) & $84(82-86)$ & $0(0-1)$ & $2(2-3)$ & $13(11-15)$ \\
\hline \multirow[t]{4}{*}{ DPT3+Hib+HBV } & Paarl & 94 (91-96) & 92 (90-93) & $1(0-3)$ & $0(0-0)$ & $6(4-9)$ \\
\hline & Umlazi & 90 (87-93) & $86(83-88)$ & $0(0-2)$ & $4(3-4)$ & $10(7-13)$ \\
\hline & Rietvlei & $74(68-80)$ & 70 (64-73) & $0(0-3)$ & $4(4-4)$ & $26(20-32)$ \\
\hline & Total & $88(86-90)$ & $85(83-86)$ & $1(0-1)$ & $3(3-3)$ & $12(10-14)$ \\
\hline
\end{tabular}

Coverage at end of follow-up and the proportion receiving the vaccinations within the recommended time periods (timely vaccination) are presented with 95\% confidence intervals. Similarly, untimely vaccination are categorised into vaccines given earlier or later than recommended. Rates given for each of the three sites and in total

* The pentavalent vaccine (DPT1+Hib+HBV) protects against diphtheria, pertussis, tetanus, Haemophilus influenzae type b and hepatitis B

The nationally reported coverage rates for the third doses of the HBV and Hib vaccines in 2004 were $92 \%$ and $93 \%$, respectively [23]. This is somewhere between the estimates for Paarl and Umlazi that is reported in this study for the third dose of the corresponding pentavalent vaccine in this study, but much higher than the coverage rates in the poor area Rietvlei.

The differences between vaccination coverage and vaccination timeliness were not as large for most vaccines in this study compared to what has been observed in some other studies [3,7-10]. This means that most of those who were vaccinated, received their vaccines within the recommended time period. Still, the proportion who received their vaccines later than recommended for the second and third vaccination visits was substantial. In general, the factors associated with good vaccination coverage were the factors that were associated with timely vaccination.

Although it is difficult to assess the timeliness and coverage of measles vaccination from this study, the trend with increasingly lower coverage for the later vaccinations compared to the earlier vaccinations, indicate that the situation for the measles vaccine is likely to be worse than for the last pentavalent and oral polio vaccines. Currently, the South African vaccination program has included the rotavirus and pneumococcal vaccines, 


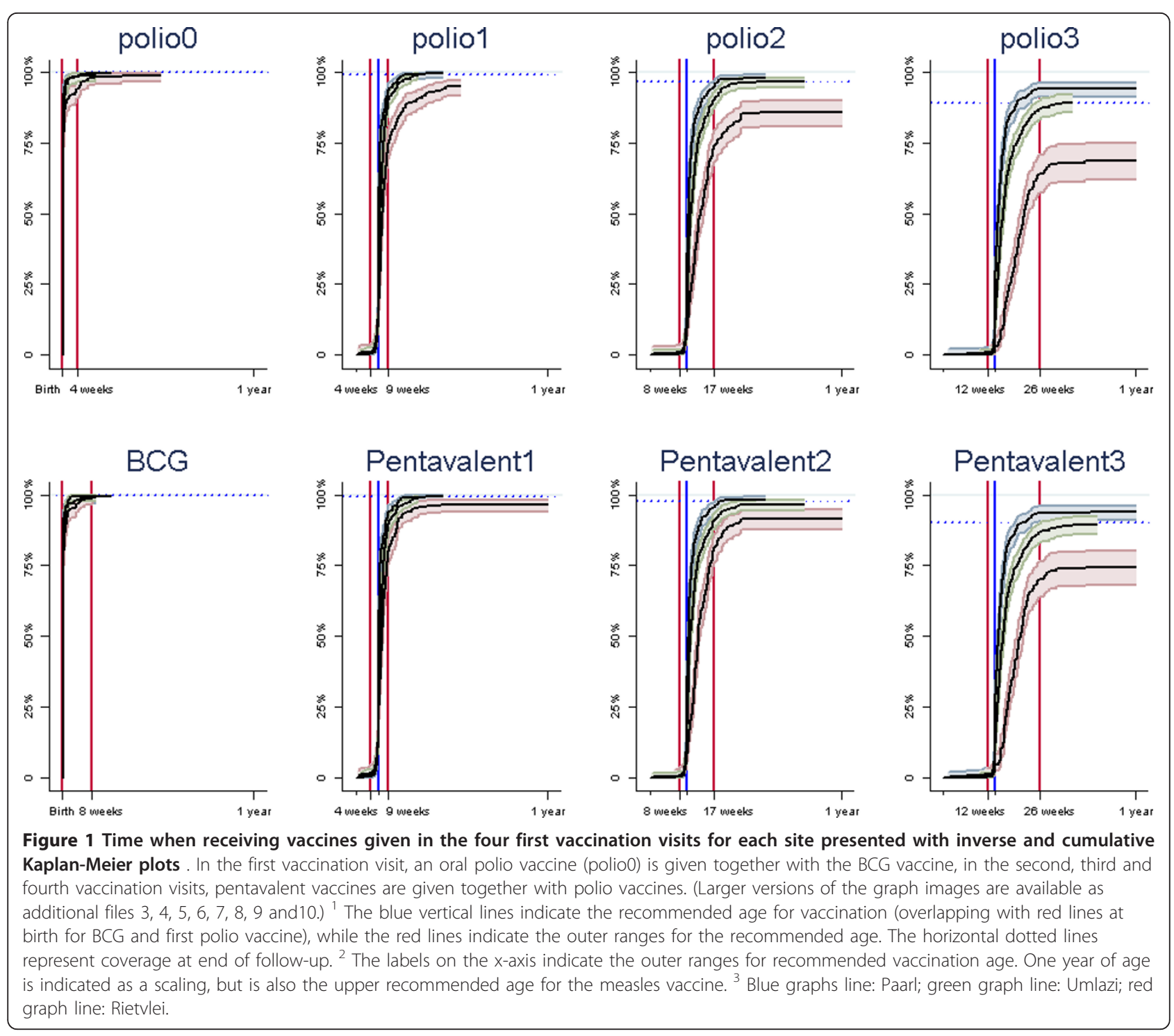

which have a potential to avert a large number of child deaths [24]. These vaccines are supposed to be given together with the pentavalent and measles vaccines. Thus, sub-optimal vaccination with for example the third pentavalent vaccine is likely to be associated with unsatisfactory vaccination also with the rotavirus and pneumococcal vaccines. To get optimal protection from these vaccines, good vaccination coverage and timeliness are essential $[25,26]$.

Globally there have been gradual improvements in vaccination coverage, with large reductions in measles, pertussis and tetanus mortality. Still, these diseases were responsible for about $4 \%$ of the global child mortality according to a systematic analysis from 2008 [15]. The same report attributed few deaths in South Africa to these diseases, with only one pertussis death, one measles death, and 139 deaths from tetanus. This is likely to be an underestimate, considering the relatively low coverage rates we observed in Rietvlei for the vaccines given earlier than the measles vaccine, in addition to large measles outbreaks in South Africa, including an extensive national outbreak reported in June 2010 [16]. More than 15000 cases with measles and 18 confirmed deaths were report from this source.

Most deaths from diseases with available vaccines are preventable, and diseases such as measles and polio can be eliminated with vaccination [27-29]. To accomplish this, a high vaccination coverage rate is needed [30,31]. The vaccination coverage in this study was inadequate particularly for the later vaccines in some of the areas, and several children were also vaccinated too late, leaving them susceptible to diseases when their maternal antibodies have declined to levels insufficient to protect them [1-3]. For some vaccines including the BCG vaccine, late 


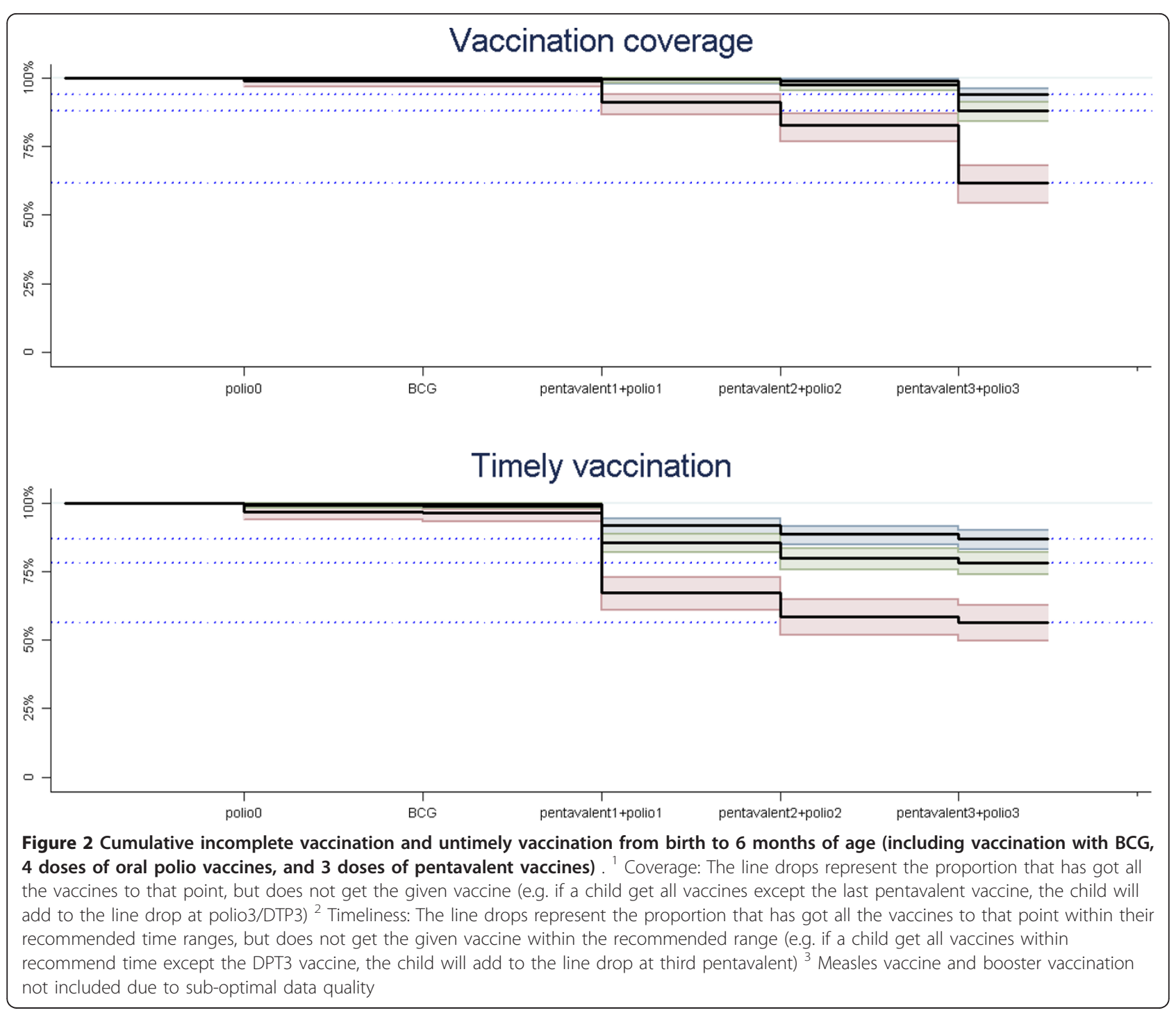

administration has been associated with higher mortality [6]. In this study, nearly all received the BCG and first oral polio vaccine soon after birth. There is also a lack of evidence on potential indirect effects of timing of immunisation outside the recommended time ranges [12].

The presence of the measles booster vaccine in the South African immunisation program offers some insurance if children are either vaccinated earlier than recommended or miss the first measles vaccine [30,32-34]. One of the associations that was found in this study was a lower risk of incomplete vaccination among those who had an intention to formula feed. This might be a reflection that many of these children have HIV-positive mothers, and they might be followed up more closely, also with respect to vaccination of their children. For children infected with HIV, the decision on when to vaccinate is further complicated as severe immune suppression is a contraindication to vaccination with live vaccines such as the measles vaccine [34]. However, those with symptomatic HIV infection but who are not severely immuno compromised are considered for vaccination [20].

There was also an association with lower risk of incomplete immunisation among children receiving breastfeeding counselling than children in the control arm. Despite the fact that this intervention did not prove particularly effective in increasing the practice of exclusive breastfeeding in South Africa (submitted) and even though the mothers were not counselled on vaccines, there was a general focus on child health in addition to the breastfeeding in the intervention arm of the study which might explain the positive impact on vaccination.

To have a good vaccination program, a well-functioning health system is required [35], where both individual and contextual factors are of importance for utilization of the health services [36]. The very substantial differences in 


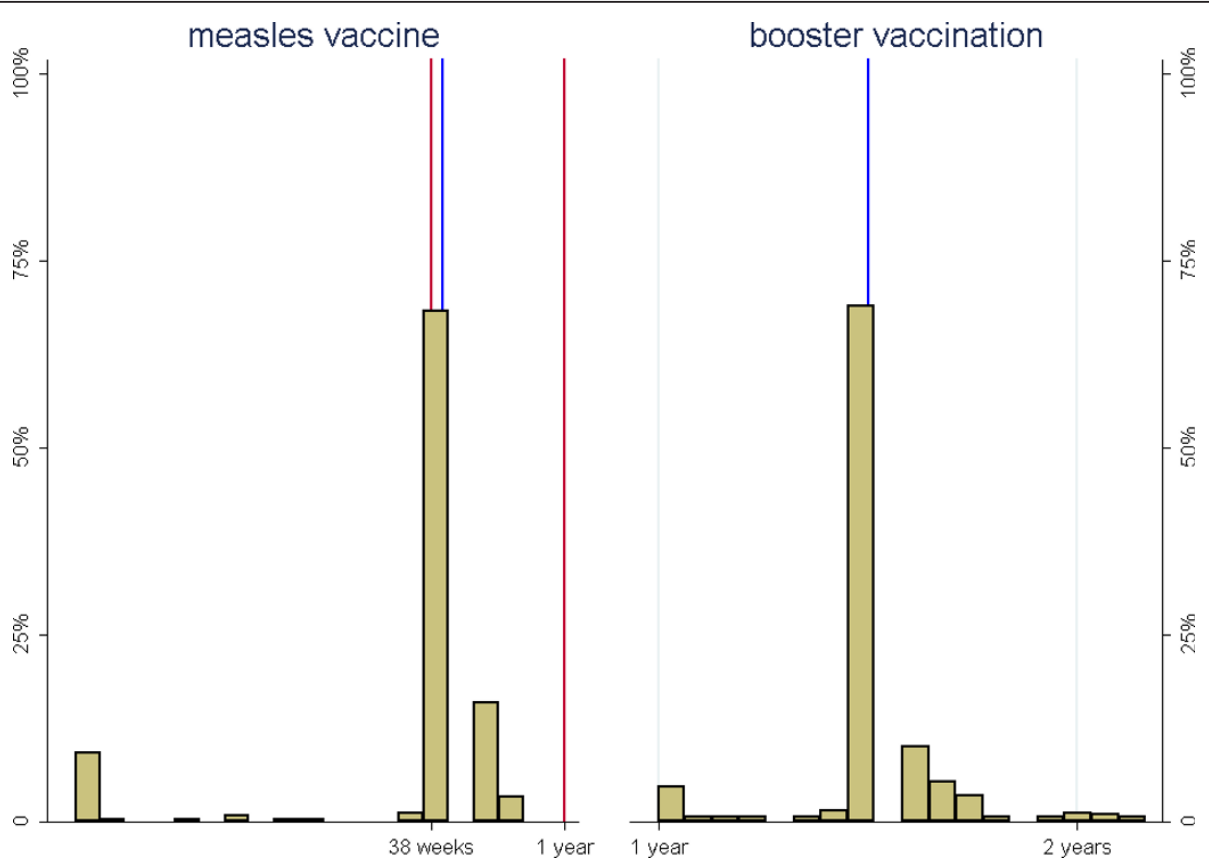

Figure 3 Time when receiving the measles vaccine and booster vaccination with measles, DPT and oral polio presented with histogram (must be interpreted with caution). ${ }^{1}$ The blue vertical lines indicate the recommended age for vaccination, while the red lines on the measles vaccine indicate the outer ranges for the recommended age. ${ }^{2}$ When the booster vaccine against measles was given at different time from the booster against polio and DPT (3 cases), the time of the measles booster will be presented.

Table 2 Baseline characteristics of study participants presented with number (n) and percentages, determinants of untimely vaccination, and determinants of incomplete vaccination at end of follow-up presented with hazard ratios (HR) with $95 \%$ confidence interval assessed with a cluster adjusted Cox regression model

\begin{tabular}{|c|c|c|c|c|c|}
\hline & $\begin{array}{c}\text { n (\%) } \\
765\end{array}$ & $\begin{array}{l}\text { HR for untimely } \\
\text { Unadjusted model }\end{array}$ & $\begin{array}{l}\text { vaccination }(95 \% \mathrm{Cl}) \\
\text { Adjusted model }\end{array}$ & $\begin{array}{l}\text { HR for incomplete } \\
\text { Unadjusted model }\end{array}$ & $\begin{array}{c}\text { vaccination }(95 \% \mathrm{Cl}) \\
\text { Adjusted model }\end{array}$ \\
\hline \multicolumn{6}{|l|}{ Mother's age } \\
\hline$\leq 19$ & $265(23)$ & 1 & & 1 & \\
\hline $20-24$ & $387(34)$ & $0.77(0.57-1.0)$ & & $0.70(0.47-1.1)$ & \\
\hline $25-29$ & $249(22)$ & $0.91(0.68-1.2)$ & & $0.59(0.41-0.85)$ & \\
\hline$\geq 30$ & $234(21)$ & $0.90(0.65-1.2)$ & & $0.96(0.66-1.4)$ & \\
\hline Marital status & & * & & * & \\
\hline Married or cohabiting & $712(63)$ & 1 & & 1 & \\
\hline Single, widowed, separated or divorced & $422(37)$ & $0.64(0.51-0.81)$ & & $0.65(0.45-0.95)$ & \\
\hline \multicolumn{6}{|l|}{ Gender of infant } \\
\hline Girl & $568(50)$ & 1 & 1 & 1 & \\
\hline Boy & $559(50)$ & $1.3(1.0-1.6)$ & $1.2(0.92-1.5)$ & $0.95(0.68-1.3)$ & \\
\hline Mother's education & & & & * & \\
\hline Primary (0-7 years) & $142(11)$ & 1 & & 1 & \\
\hline Secondary (8-11 years) & $756(59)$ & $1.2(0.80-1.7)$ & & $0.62(0.40-0.96)$ & \\
\hline Higher (12 or above) & $378(30)$ & $0.81(0.53-1.2)$ & & $0.31(0.19-0.53)$ & \\
\hline Socio economic wealth index & & * & & * & \\
\hline Least poor quintile & $234(21)$ & 1 & & 1 & \\
\hline $4^{\text {th }}$ quintile & $253(22)$ & $0.91(0.56-1.5)$ & & $1.4(0.70-3.0)$ & \\
\hline $3^{\text {rd }}$ quintile & $223(20)$ & $1.1(0.69-1.8)$ & & $2.2(1.1-4.4)$ & \\
\hline $2^{\text {nd }}$ quintile & $210(18)$ & $1.5(1.0-2.4)$ & & $3.6(1.8-7.0)$ & \\
\hline Poorest quintile & $217(19)$ & $2.4(1.6-3.6)$ & & $7.5(4.0-14)$ & \\
\hline
\end{tabular}


Table 2 Baseline characteristics of study participants presented with number (n) and percentages, determinants of untimely vaccination, and determinants of incomplete vaccination at end of follow-up presented with hazard ratios (HR) with 95\% confidence interval assessed with a cluster adjusted Cox regression model (Continued)

Living area

Paarl

Rietvlei

Umlazi

Place of delivery

Home

In health facility

Health councelling

Normal

Additional peer-counselling

Intention to formula feed

Number of siblings

None

1 - 2

3 or above

Mother's body mass index (BMI)

$$
<20
$$

$20-24.9$

$25-29.9$

$\geq 30$

$\begin{array}{lc}537(47) & 1 \\ 464(41) & 1.0(0.84-1.3) \\ 130(11) & 1.4(1.08-1.9)\end{array}$

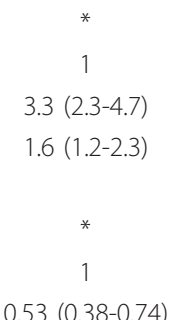

$0.53(0.38-0.74)$

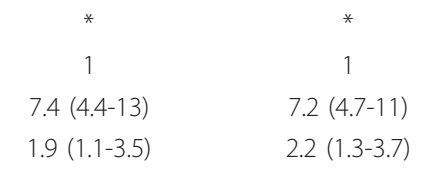

*

1

$0.36(0.20-0.64)$

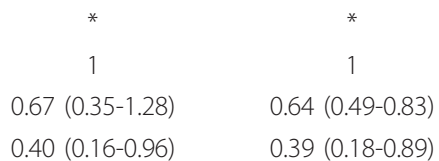

$\begin{array}{ll}535(17) & 0.74(0.41-1.3)\end{array}$

$0.40(0.16-0.96)$

$0.39(0.18-0.89)$

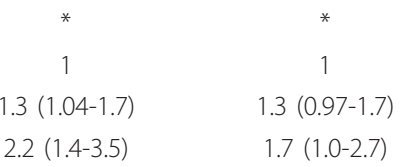

* Significantly associated variables (both categorised and socio-economy also uncategorised).

Interactions were checked and none were considered meaningful to include.

All factors are cluster adjusted.

vaccination coverage between the three sites in this study are largely a reflection of the relative strengths and functionality of the health systems in these three areas, as well as the large differences in socio economic status [37]. Achieving good and timely vaccination coverage and eliminating the vast disparities between the different geographical areas will require both general developmental interventions - concerning education, infrastructure and communications - as well as health system strengthening. The latter efforts will need to focus on good coverage by health services, particularly at community and primary levels with effective referral mechanisms, well-equipped and organised clinics with short waiting times and effective integration of maternal and child health activities, as well as good media promotion and campaigns [35,38]. Vaccination reminders are an effective strategy to improve vaccination, particularly phone call reminders [39]. The feasibility of instituting vaccination reminders should be explored, given that possession and use of cell phones in South Africa has increased dramatically in the recent past.

This study has some limitations. The time estimate of the first measles vaccination is likely to be biased to vaccination before 12 months because a question from the last interview was missed out. Thus, the time distribution of the measles vaccination must be interpreted with caution. Some of the last follow-up interviews were also done too early to assess the booster vaccination, but this is likely to have affected the estimates less than for the first measles vaccine.

The children who died during follow-up might have had different vaccination status compared to the surviving majority [40], but mortality was low and therefore this is unlikely to have biased our estimates. Most of the vaccination information was collected in the 24 weeks interview and those who were not interviewed at that point and did not provide sufficient vaccination information in the other interviews visits might also have had a different vaccination status. The proportion of participants with insufficient information to be assessed was 13\%. These participants were more often from the poorer areas, and thus the combined estimates might be slightly lower than what has been presented in this paper. The timeliness of vaccination in the few children who had immunisation indicated as received but not dated in the 
health card could be different from the majority where it was dated. As there were only a few and that they had similar baseline characteristics (data not shown), we believe this has not biased our estimates markedly. Contraindications for vaccination were not assessed [41], but this is relevant only in a few cases. It may be justified to temporarily postpone vaccination in some few cases when children are moderately or severely ill. Some children may have been HIV-positive with severe immune suppression, which is a contraindication for live vaccines such as the measles vaccine and complicates the assessment of coverage and timeliness [34,41]]. In this study, many of the mothers in the intention to formula feed arm were HIV-positive, but the number of infected children is likely to be much less. As the Cox regression model assessed timeliness with specific accepted time ranges, there were several ties with equal time-to-event. To improve model robustness, we used the exact partiallikelihood method for handling ties. The study also has several strengths compared to many studies assessing vaccination; these include a prospective design, date assessment of the vaccines, assessment of all recommended vaccines, inclusion of different sites, and the use of time-to-event analysis.

\section{Conclusion}

There are substantial differences in vaccination coverage and timely vaccination between different geographical areas in South Africa. There was a trend towards worse vaccination coverage with the later vaccines in the vaccination programme. To have adequate disease protection and to aim for elimination of some of the diseases, there is a need for continued efforts to improve vaccination coverage and timeliness.

There is also a need for efforts and research to assess whether general socio economic development as well as strengthening of the health systems could improve vaccination coverage and reduce disparities between different geographical areas and socio economic groups.

\section{Additional material}

Additional file 1: Figure S1: Study profile. Number of participants in the different trial arms: Good Start Study (intention to formula feed) and PROMISE-EBF study (intention to breastfeed) with its intervention and control arms. The number of participants interviewed in the different visits is given with proportion of the included participants in the parenthesis. Cumulative proportion of lost-to-follow-up is also added in brackets.

Additional file 2: Figure S2: Figure presenting age distribution when the different vaccines are given for all the sites combined (hazard plot). ${ }^{1}$ The blue vertical lines indicate the recommended age for vaccination (overlapping with red lines at birth for BCG and first polio vaccine), while the red lines indicate the outer ranges for the

recommended age. The horizontal dotted lines represent coverage at end of follow-up. ${ }^{2}$ The labels on the $x$-axis indicate the outer ranges for recommended vaccination age. One year of age is indicated as a scaling, but is also the upper recommended age for the measles vaccine.

Additional file 3: Figure S3: Timing of the oral polio vaccine (polio0) given at birth for each site presented with Kaplan-Meier plots (inverse and cumulative). ${ }^{1}$ The blue vertical lines indicate the recommended age for vaccination (overlapping with red lines at birth for BCG and first polio vaccine), while the red lines indicate the outer ranges for the recommended age. The horizontal dotted lines represent coverage at end of follow-up. ${ }^{2}$ The labels on the $x$-axis indicate the outer ranges for recommended vaccination age. One year of age is indicated as a scaling, but is also the upper recommended age for the measles vaccine. ${ }^{3}$ Blue graphs line: Paarl; green graph line: Umlazi; red graph line: Rietvlei.

Additional file 4: Figure S4: Timing of the BCG vaccine given at birth for each site presented with Kaplan-Meier plots (inverse and cumulative). ${ }^{1}$ The blue vertical lines indicate the recommended age for vaccination (overlapping with red lines at birth for BCG and first polio vaccine), while the red lines indicate the outer ranges for the

recommended age. The horizontal dotted lines represent coverage at end of follow-up. ${ }^{2}$ The labels on the $x$-axis indicate the outer ranges for recommended vaccination age. One year of age is indicated as a scaling, but is also the upper recommended age for the measles vaccine. ${ }^{3}$ Blue graphs line: Paarl; green graph line: Umlazi; red graph line: Rietvlei.

Additional file 5: Figure S5: Timing of the second oral polio vaccine (polio1) for each site presented with Kaplan-Meier plots (inverse and cumulative). ${ }^{1}$ The blue vertical lines indicate the recommended age for vaccination (overlapping with red lines at birth for BCG and first polio vaccine), while the red lines indicate the outer ranges for the recommended age. The horizontal dotted lines represent coverage at end of follow-up. ${ }^{2}$ The labels on the $x$-axis indicate the outer ranges for recommended vaccination age. One year of age is indicated as a scaling, but is also the upper recommended age for the measles vaccine. ${ }^{3}$ Blue graphs line: Paarl; green graph line: Umlazi; red graph line: Rietvlei.

Additional file 6: Figure S6: Timing of the first pentavalent vaccine (pentavalent1) for each site presented with Kaplan-Meier plots (inverse and cumulative). ${ }^{1}$ The blue vertical lines indicate the recommended age for vaccination (overlapping with red lines at birth for BCG and first polio vaccine), while the red lines indicate the outer ranges for the recommended age. The horizontal dotted lines represent coverage at end of follow-up. ${ }^{2}$ The labels on the $x$-axis indicate the outer ranges for recommended vaccination age. One year of age is indicated as a scaling, but is also the upper recommended age for the measles vaccine. ${ }^{3}$ Blue graphs line: Paarl; green graph line: Umlazi; red graph line: Rietvlei.

Additional file 7: Figure S7: Timing of the third oral polio vaccine (polio2) for each site presented with Kaplan-Meier plots (inverse and cumulative). ${ }^{1}$ The blue vertical lines indicate the recommended age for vaccination (overlapping with red lines at birth for BCG and first polio vaccine), while the red lines indicate the outer ranges for the recommended age. The horizontal dotted lines represent coverage at end of follow-up. ${ }^{2}$ The labels on the $x$-axis indicate the outer ranges for recommended vaccination age. One year of age is indicated as a scaling, but is also the upper recommended age for the measles vaccine. ${ }^{3}$ Blue graphs line: Paarl; green graph line: Umlazi; red graph line: Rietvlei.

Additional file 8: Figure S8: Timing of the second pentavalent vaccine (pentavalent2) for each site presented with Kaplan-Meier plots (inverse and cumulative). ${ }^{1}$ The blue vertical lines indicate the recommended age for vaccination (overlapping with red lines at birth for BCG and first polio vaccine), while the red lines indicate the outer ranges for the recommended age. The horizontal dotted lines represent coverage at end of follow-up. ${ }^{2}$ The labels on the $x$-axis indicate the outer ranges for recommended vaccination age. One year of age is indicated as a scaling, but is also the upper recommended age for the measles vaccine. ${ }^{3}$ Blue graphs line: Paarl; green graph line: Umlazi; red graph line: Rietvlei.

Additional file 9: Figure 59: Timing of the fourth oral polio vaccine (polio3) for each site presented with Kaplan-Meier plots (inverse and cumulative). ${ }^{1}$ The blue vertical lines indicate the recommended 
age for vaccination (overlapping with red lines at birth for BCG and first polio vaccine), while the red lines indicate the outer ranges for the recommended age. The horizontal dotted lines represent coverage at end of follow-up. ${ }^{2}$ The labels on the $x$-axis indicate the outer ranges for recommended vaccination age. One year of age is indicated as a scaling, but is also the upper recommended age for the measles vaccine. ${ }^{3}$ Blue graphs line: Paarl; green graph line: Umlazi; red graph line: Rietvlei.

Additional file 10: Figure S10: Timing of the third pentavalent vaccine (pentavalent3) for each site presented with Kaplan-Meier plots (inverse and cumulative). ${ }^{1}$ The blue vertical lines indicate the recommended age for vaccination (overlapping with red lines at birth for BCG and first polio vaccine), while the red lines indicate the outer ranges for the recommended age. The horizontal dotted lines represent coverage at end of follow-up. ${ }^{2}$ The labels on the $x$-axis indicate the outer ranges for recommended vaccination age. One year of age is indicated as a scaling, but is also the upper recommended age for the measles vaccine. ${ }^{3}$ Blue graphs line: Paarl; green graph line: Umlazi; red graph line: Rietvlei.

\section{Abbreviations}

BCG: Bacillus Calmette-Guérin; Cl: confidence interval; DTP: diphtheria, tetanus and pertussis; EPI: Expanded Programme on Immunization; HR: hazard ratio; HBV: hepatitis B; HiB: Haemophilus influenza type B; WHO: World Health Organisation.

\section{Acknowledgements}

We thank the data collectors, all the families who contributed to this study, and Johnny Nare Rakgole, Zainab Waggie and Charles Shey Wiysonge for information about the South African EPI.

Thanks also to the PROMISE-EBF Study Group:

Steering Committee: Thorkild Tylleskär, Philippe van de Perre, Eva-Charlotte Ekström, Nicolas Meda, James K Tumwine, Chipepo Kankasa, Debra Jackson Participating countries and investigators:

Norway: Thorkild Tylleskär, Ingunn MS Engebretsen, Lars T Fadnes, Eli Fjeld Falnes, Knut Fylkesnes, Jørn Klungsøyr, Anne Nordrehaug-Åstrøm, Øystein Evjen Olsen, Bjarne Robberstad, Halvor Sommerfelt

France: Philippe Van de Perre

Sweden: Eva-Charlotte Ekström, Barni Nor

Burkina Faso: Nicolas Meda, Hama Diallo, Thomas Ouedrago, Jerem

Rouamba, Bernadette Traoré Germain Traoré, Emmanuel Zabsonré

Uganda: James K. Tumwine, Caleb Bwengye, Charles Karamagi, Victoria

Nankabirwa, Jolly Nankunda, Grace Ndeezi, Margaret Wandera

Zambia: Chipepo Kankasa, Mary Katepa-Bwalya, Chafye Siuluta, Seter Siziya

South Africa: Debra Jackson, Mickey Chopra, Mark Colvin, Tanya Doherty, Carl Lombard, Ameena E Goga, Lungiswa Nkonki, David Sanders, Sonja

Swanevelder, Wanga Zembe

(Country PI first, others in alphabetical order of surname)

\section{Funding}

The study was part of the European Union-funded project PROMISE-EBF (contract no INCO-CT 2004-003660, http://www.promiseresearch.org). It was also financially supported through the Swedish/South Africa Cooperation and the South African National Research Foundation (NRF) and Centers for Disease Control \& Prevention South Africa/PEPFAR.

LTF, IMSE, HS and TT were employed and funded by the University of Bergen. WZ was employed and funded by Medical Research Council, South Africa. DS and DJ were employed and funded by University of the Western Cape. The funders had no role in study design, data collection and analysis, decision to publish, or preparation of the manuscript.

\section{Author details}

${ }^{1}$ Centre for International Health, University of Bergen, Norway. ${ }^{2}$ School of Public Health, University of Western Cape, South Africa. ${ }^{3}$ Medical Research Council, South Africa. ${ }^{4}$ Division of Infectious Disease Control, Norwegian Institute of Public Health, Norway.

\section{Authors' contributions}

LTF: design, analysis and writing. DJ, WZ, DS: design, implementation and Co-writing. IMSE, HS, TT: design, analysis and co-writing. The PROMISE-EBF
Study Group: design and implementation. All authors read and approved the final manuscript.

\section{Competing interests}

The authors declare that they have no competing interests.

Received: 18 February 2011 Accepted: 27 May 2011

Published: 27 May 2011

\section{References}

1. Grant CC, Roberts M, Scragg R, Stewart J, Lennon D, Kivell D, Ford R, Menzies R: Delayed immunisation and risk of pertussis in infants: unmatched case-control study. Brit Med J 2003, 326(7394):852-853.

2. Kolos V, Menzies R, Mclntyre P: Higher pertussis hospitalization rates in indigenous Australian infants, and delayed vaccination. Vaccine 2007, 25(4):588-590

3. Heininger $U$, Zuberbuhler M: Immunization rates and timely administration in pre-school and school-aged children. Eur J Pediatr 2006, 165(2):124-129.

4. von Kries R, Bohm O, Windfuhr A: Haemophilus influenzae b-vaccination: the urgency for timely vaccination. Eur J Pediatr 1997, 156(4):282-287.

5. The measles epidemic. The problems, barriers, and recommendations. The National Vaccine Advisory Committee. JAMA 1991, 266(11):1547-1552

6. Breiman RF, Streatfield PK, Phelan M, Shifa N, Rashid M, Yunus M: Effect of infant immunisation on childhood mortality in rural Bangladesh: analysis of health and demographic surveillance data. Lancet 2004, 364(9452):2204-2211.

7. Akmatov MK, Kretzschmar M, Kramer A, Mikolajczyk RT: Timeliness of vaccination and its effects on fraction of vaccinated population. Vaccine 2008, 26(31):3805-3811.

8. Dayan GH, Shaw KM, Baughman AL, Orellana LC, Forlenza R, Ellis A, Chaui J, Kaplan S, Strebel P: Assessment of delay in age-appropriate vaccination using survival analysis. Am J Epidemiol 2006, 163(6):561-570.

9. Clark A, Sanderson C: Timing of children's vaccinations in 45 low-income and middle-income countries: an analysis of survey data. Lancet 2009, 373(9674):1543-1549.

10. Luman ET, Barker LE, Shaw KM, McCauley MM, Buehler JW, Pickering LK: Timeliness of childhood vaccinations in the United States - Days undervaccinated and number of vaccines delayed. Jama-J Am Med Assoc 2005, 293(10):1204-1211.

11. Fadnes $L T$, Nankabirwa V, Sommerfelt $H$, Tylleskar T, Tumwine JK, Engebretsen IM: Is vaccination coverage a good indicator of ageappropriate vaccination? A prospective study from Uganda. Vaccine 2011, 29(19):3564-3570.

12. Shann F, Nohynek H, Scott JA, Hesseling A, Flanagan KL: Randomized trials to study the nonspecific effects of vaccines in children in low-income countries. Pediatr Infect Dis / 2010, 29(5):457-461.

13. Aaby $P$, Rodrigues $A$, Biai $S$, Martins $C$, Veirum JE, Benn $C S$, Jensen $H$ : Oral polio vaccination and low case fatality at the paediatric ward in Bissau, Guinea-Bissau. Vaccine 2004, 22(23-24):3014-3017.

14. Ndiritu M, Cowgill KD, Ismail A, Chiphatsi S, Kamau T, Fegan G, Feikin DR, Newton CR, Scott JA: Immunization coverage and risk factors for failure to immunize within the Expanded Programme on Immunization in Kenya after introduction of new Haemophilus influenzae type $b$ and hepatitis b virus antigens. BMC Public Health 2006, 6(132).

15. Black RE, Cousens S, Johnson HL, Lawn JE, Rudan I, Bassani DG, Jha P, Campbell H, Walker CF, Cibulskis R, et al: Global, regional, and national causes of child mortality in 2008: a systematic analysis. Lancet 2010, 375(9730):1969-1987.

16. WHO and UNICEF concerned about measles outbreak in Eastern and Southern Africa. [http://www.unicef.org/media/media_54018.html].

17. Nankunda J, Tylleskar T, Ndeezi G, Semiyaga N, Tumwine JK: Establishing individual peer counselling for exclusive breastfeeding in Uganda: implications for scaling-up. Matern Child Nutr 2010, 6(1):53-66.

18. National Department of Health South Africa: The national HIV and syphilis prevalence survey South Africa 2007. Pretoria, South Africa: National Department of Health 2007.

19. Jackson DJ, Chopra M, Doherty TM, Colvin MS, Levin JB, Willumsen JF, Goga AE, Moodley P: Operational effectiveness and 36 week HIV-free 
survival in the South African programme to prevent mother-to-child transmission of HIV-1. AIDS 2007, 21(4):509-516.

20. The South African EPI (EPI-SA) schedule and method of vaccine administration. [http://www.savic.ac.za].

21. Laubereau B, Hermann M, Schmitt HJ, Weil J, von Kries R: Detection of delayed vaccinations: a new approach to visualize vaccine uptake. Epidemiol Infect 2002, 128(2):185-192.

22. Howe LD, Hargreaves JR, Huttly SR: Issues in the construction of wealth indices for the measurement of socio-economic position in low-income countries. Emerg Themes Epidemiol 2008, 5:3.

23. Arevshatian L, Clements C, Lwanga S, Misore A, Ndumbe P, Seward J, Taylor P: An evaluation of infant immunization in Africa: is a transformation in progress? Bull World Health Organ 2007, 85(6):449-457.

24. World Health Organization: State of the world's vaccines and immunization., 32009 [http://www.who.int/immunization/sowvi/en/].

25. Smith PJ, Nuorti JP, Singleton JA, Zhao Z, Wolter KM: Effect of vaccine shortages on timeliness of pneumococcal conjugate vaccination: results from the 2001-2005 National Immunization Survey. Pediatrics 2007, 120(5):e1165-1173.

26. Daskalaki I, Spain CV, Long SS, Watson B: Implementation of rotavirus immunization in Philadelphia, Pennsylvania: high levels of vaccine ineligibility and off-label use. Pediatrics 2008, 122(1):e33-38.

27. Biellik R, Madema S, Taole A, Kutsulukuta A, Allies E, Eggers R, Ngcobo N, Nxumalo M, Shearley A, Mabuzane $E$, et al: First 5 years of measles elimination in southern Africa: 1996-2000. Lancet 2002, 359(9317):1564-1568.

28. Sudfeld CR, Navar AM, Halsey NA: Effectiveness of measles vaccination and vitamin A treatment. Int J Epidemiol 2010, 39(Suppl 1):i48-55.

29. Polio vaccines and polio immunization in the pre-eradication era: WHO position paper. Wkly Epidemiol Rec 2010, 85(23):213-228.

30. Orenstein WA, Strebel PM, Papania M, Sutter RW, Bellini WJ, Cochi SL: Measles eradication: is it in our future? Am J Public Health 2000, 90(10):1521-1525.

31. Stittelaar KJ, de Swart RL, Osterhaus AD: Vaccination against measles: a neverending story. Expert Rev Vaccines 2002, 1(2):151-159.

32. Dai B, Chen ZH, Liu QC, Wu T, Guo CY, Wang XZ, Fang HH, Xiang YZ: Duration of immunity following immunization with live measles vaccine: 15 years of observation in Zhejiang Province, China. Bull World Health Organ 1991, 69(4):415-423.

33. Strebel P, Cochi S, Grabowsky M, Bilous J, Hersh BS, Okwo-Bele JM, Hoekstra E, Wright P, Katz S: The unfinished measles immunization agenda. J Infect Dis 2003, 187(Suppl 1):S1-7.

34. World Health Organization: Measles Vaccines: WHO Position Paper. Weekly epidemiological record 2009, 84:349-360.

35. Rwashana AS, Williams DW, Neema S: System dynamics approach to immunization healthcare issues in developing countries: a case study of Uganda. Health Informatics J 2009, 15(2):95-107.

36. Andersen RM: National health surveys and the Behavioral Model of Health Services Use. Med Care 2008, 46(7):647-653.

37. Coovadia H, Jewkes R, Barron P, Sanders D, Mclntyre D: The health and health system of South Africa: historical roots of current public health challenges. Lancet 2009, 374(9692):817-834.

38. Ndiritu M, Cowgill KD, Ismail A, Chiphatsi S, Kamau T, Fegan G, Feikin DR, Newton CRJC, Scott JAG: Immunization coverage and risk factors for failure to immunize within the Expanded Programme on Immunization in Kenya after introduction of new Haemophilus influenzae type $b$ and hepatitis b virus antigens. BMC Public Health 2006, 6.

39. Jacobson VJ, Szilagyi P: Patient reminder and patient recall systems to improve immunization rates. Cochrane Database Syst Rev 2005, CD003941(3).

40. Jensen H, Benn CS, Lisse IM, Rodrigues A, Andersen PK, Aaby P: Survival bias in observational studies of the impact of routine immunizations on childhood survival. Trop Med Int Health 2007, 12(1):5-14.

41. Kroger AT, Atkinson WL, Marcuse EK, Pickering LK: General recommendations on immunization: recommendations of the Advisory Committee on Immunization Practices (ACIP). MMWR Recomm Rep 2006, 55(RR-15): 1-48.

\section{Pre-publication history}

The pre-publication history for this paper can be accessed here: http://www.biomedcentral.com/1471-2458/11/404/prepub

doi:10.1186/1471-2458-11-404

Cite this article as: Fadnes et al:: Vaccination coverage and timeliness in three South African areas: a prospective study. BMC Public Health 2011

11:404.

\section{Submit your next manuscript to BioMed Central and take full advantage of:}

- Convenient online submission

- Thorough peer review

- No space constraints or color figure charges

- Immediate publication on acceptance

- Inclusion in PubMed, CAS, Scopus and Google Scholar

- Research which is freely available for redistribution 